\title{
Origin of a Complex Light-Scattering Pattern from Some Crystalline Polymer Films*
}

\author{
Takeji Hashimoto, Yukinobu Murakami**, Yoshio OKamori***, \\ and Hiromichi KaWAI \\ Department of Polymer Chemistry, Faculty of Engineering, \\ Kyoto University, Kyoto 606, Japan.
}

(Received April 30, 1974)

\begin{abstract}
There is occasionally observed in some crystalline polymeric films a complex light-scattering pattern which simultaneously exhibits spherulitic scattering at small scattering angles and rod-like scattering at high scattering angles. The complex patterns which have been observed for films of poly(tetrafluoroethylene), poly(chlorotrifluoroethylene), polypropylene-polyethylene A-B-type block copolymer, and poly(ethylene oxide)polyisoprene $\mathbf{A}-\mathbf{B}$ and $\mathbf{A}-\mathbf{B}-\mathbf{A}$ block copolymers are analyzed in terms of spherulites having internal disorder with respect to the optic-axis twist-angle $\eta$. The shape of the rod-like scattering is shown to depend upon the disorder and the orientation angle $\chi$ of the optic axis with respect to spherulite radii and to be associated with fine structure of the spherulites.
\end{abstract}

KEY WORDS Light Scattering / Polymer Films / Spherulitic Scattering / Rod-like Scattering / Complex Pattern / Poly(tetrafluoroethylene) / Poly(chlorotrifluoroethylene) / Polypropylene-Polyethylene Block Copolymers / Poly(ethylene oxide)-Polyisoprene Block Copolymers / Disordered Spherulite Theory /

A light scattering method has been developed to investigate crystalline superstructures occurring in polymer films. Typical scattering patterns which are frequently found in the films are those from spherulitic and fibrillar crystalline superstructures.

In Figure 1 are shown $H_{V}$ (taken under crosspolarizers) and $V_{\nabla}$ (taken under parallel-polarizers) scattering patterns typical to spherulitic structures for a medium-density polyethylene film. The $H_{\mathrm{V}}$ scattering depends upon spherulite birefringence and gives the familiar four-leafclover-type pattern with its maximum intensity at odd multiples of the azimuthal angle $(\mu)$ of $45^{\circ}$ and at a particular scattering angle $\left(\theta_{\max }\right)$ which is inversely related to the size of the

\footnotetext{
* Published in part in Rept. Progr. Polym. Phys. Japan, 16, 1973.

** Present Address: Central Research Laboratories, Mitsubishi Chemical Industries, Inc., Hisamoto-Kamoicho, Kawasaki, Japan.

*** Present address: The Toyo Rubber Industry Co., Ltd., Inami-cho, Hyogo-ken, Japan.
}

spherulite. ${ }^{1}$ On the other hand the $V_{\mathrm{V}}$ scattering also depends upon the average refractive index of the spherulite. Theories of spherulitic scattering have been developed based upon a model of a homogeneous anisotropic sphere ${ }^{1,2}$ or circular sheet $^{3,4}$ with different radial and tangential refractive indices, embedded in the isotropic medium. This approach has successfully described the important features of most experimental patterns from spherulitic systems.

In Figure 2 are shown typical $H_{\nabla}$ and $V_{\nabla}$ patterns for the fibrillar structures of a poly(tetrafluoroethylene) (PTFE) film prepared by annealing the PTFE dispersions. A fundamental difference between the two types of patterns appears in the fact that the scattered intensity in Figure 2 decreases continuously with increasing scattering angle $(\theta)$, while that in Figure 1 passes through a maximum with increasing $\theta$.

The important features of the observed scattering from such fibrillar structures, designated as rod-like scattering, have been theoretically explained in terms of random assembly of an- 
Origin of a Complex Light-Scattering Pattern

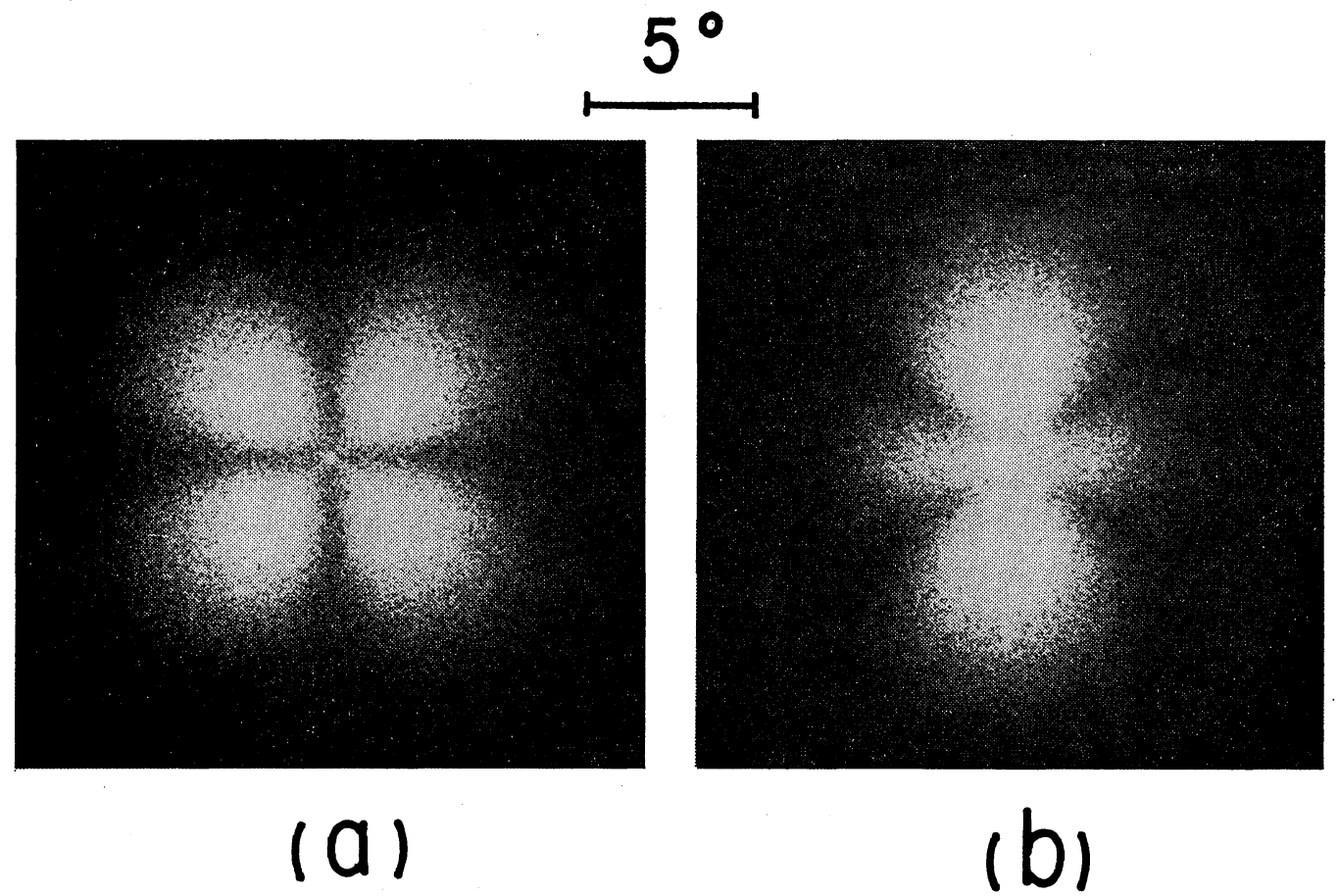

Figure 1. The $H_{\mathrm{v}}$ (a) and $V_{\mathrm{v}}$ (b) scattering patterns of a medium-density polyethylene film.

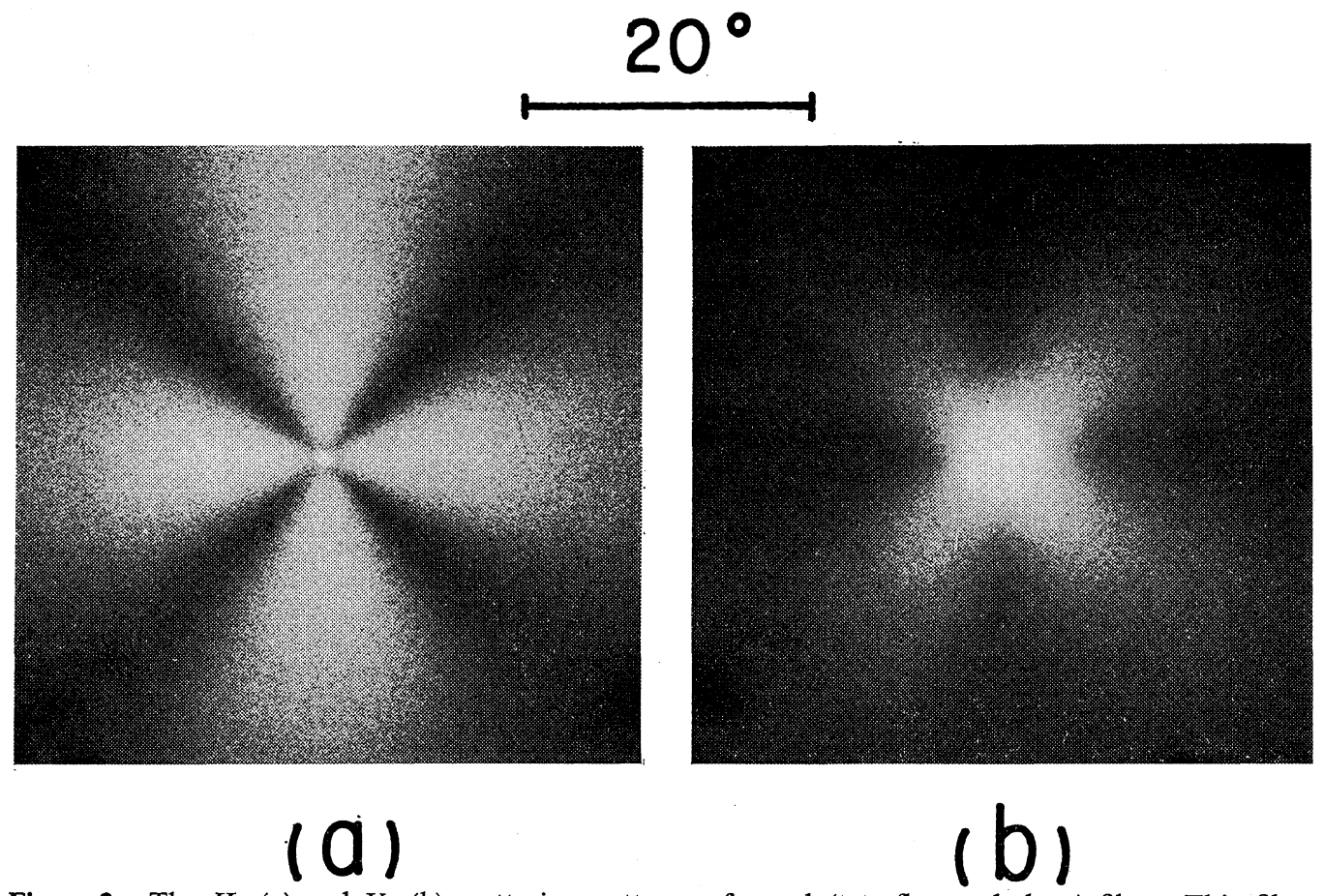

Figure 2. The $H_{\mathrm{v}}$ (a) and $V_{\mathrm{v}}$ (b) scattering patterns of a poly(tetrafluoroethylene) film. Thin films of about $30-\mu \mathrm{m}$ thick were prepared from a poly(tetrafluoroethylene) dispersion. The dispersion was annealed at $465^{\circ} \mathrm{C}$ for $2 \mathrm{hr}$ and subsequently cooled down to room temperature at a rate of $15^{\circ} \mathrm{C} / \mathrm{hr}$. 


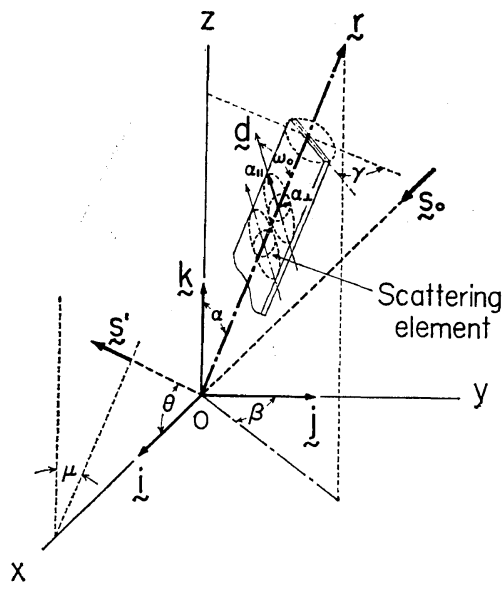

Figure 3. The model explaining the rod-like scattering. isotropic rods or sheets with their principal optic axes inclined by an angle $\omega_{0}$ with respect to the length direction of the rods $\mathrm{s}^{5-10}$ or direction normal to the sheets. ${ }^{11}$ The shape of the rod-like scattering is shown to depend upon the optic axis orientation of the scattering elements within the rods or sheets. Two types of the fibrils are considered": (i) the "fibrils of Model A" in which the orientation of the optic axis (whose unit vector is defined by $\boldsymbol{d}$ ) is constrained to a special plane within the fibrils but makes a polar angle $\omega_{0}$ with respect to the length direction of the rods, and (ii) the "fibrils of Model B" in which the vector $\boldsymbol{d}$ is arranged in cylindrical symmetry with a constant values of $\omega_{0}$ within the fibrils (see Figure 3).

The fibrils of Model B are shown to give rise to the $\times$-type or circular-type $H_{\mathrm{V}}$ scattering

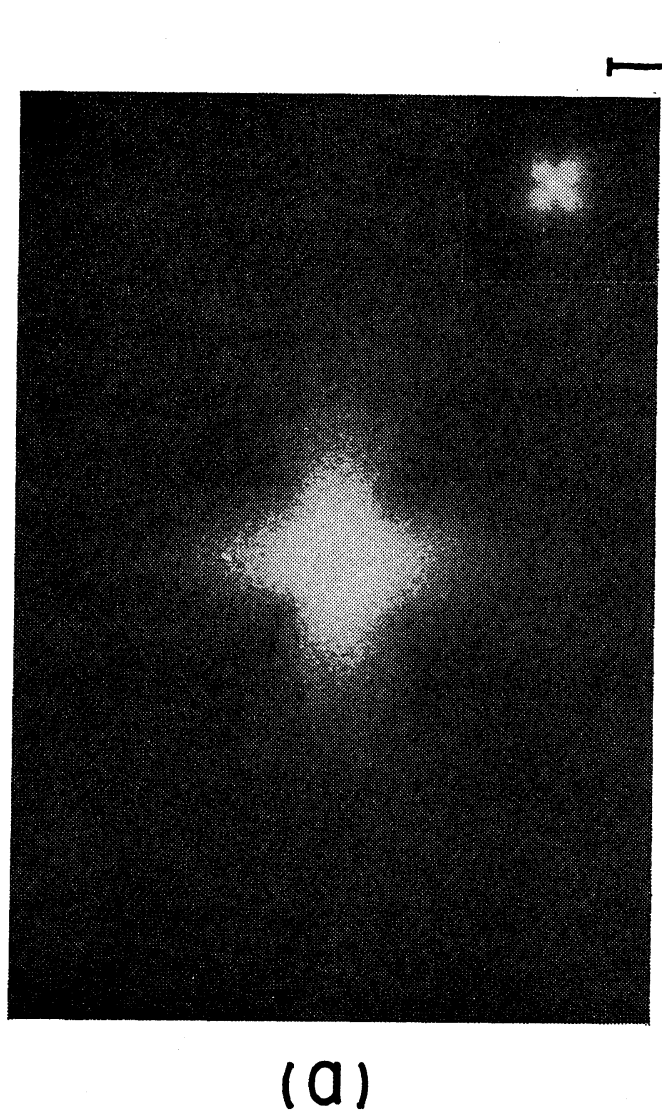

$5^{\circ}$

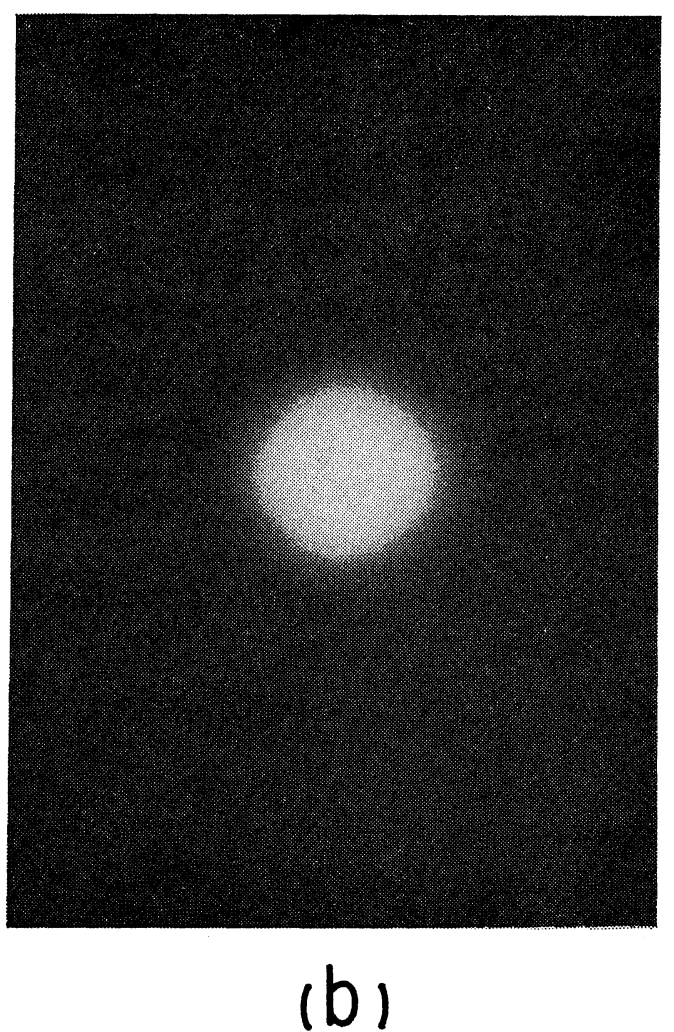

Figure 4. The $H_{\mathrm{v}}$ (a) and $V_{\mathrm{v}}$ (b) scattering patterns of a poly(tetrafluoroethylene) film. The spherulitic scattering pattern which has degenerated at the center of the +-type rod-like scattering pattern (a) is shown at upper right corner of the figure. 


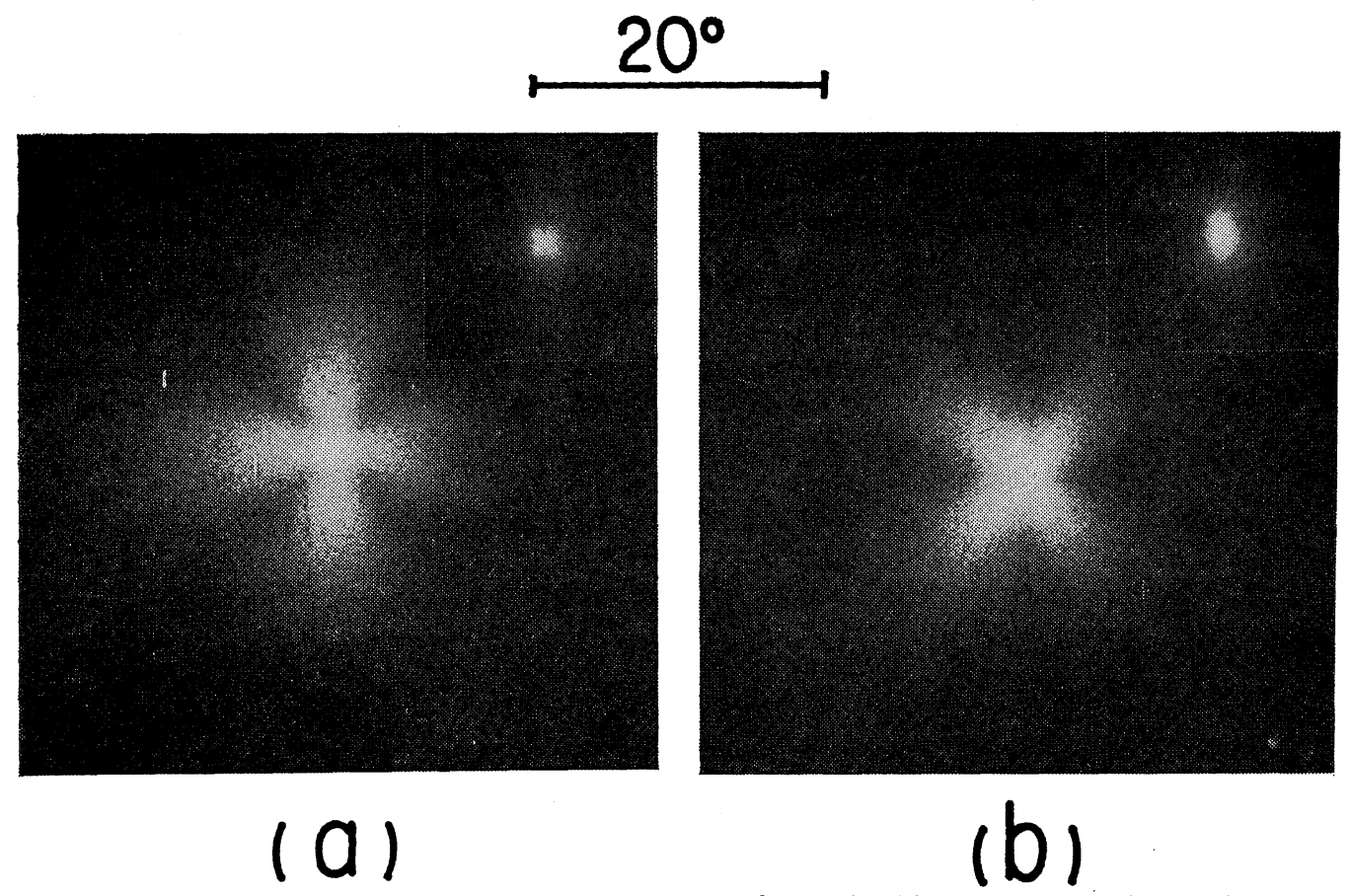

Figure 5. The $H_{\mathrm{v}}$ (a) and $V_{\mathrm{v}}$ (b) scattering patterns of a poly(chlorotrifluoroethylene) film. The overexposed spherulitic scattering patterns are shown at the upper right corner of each figure.
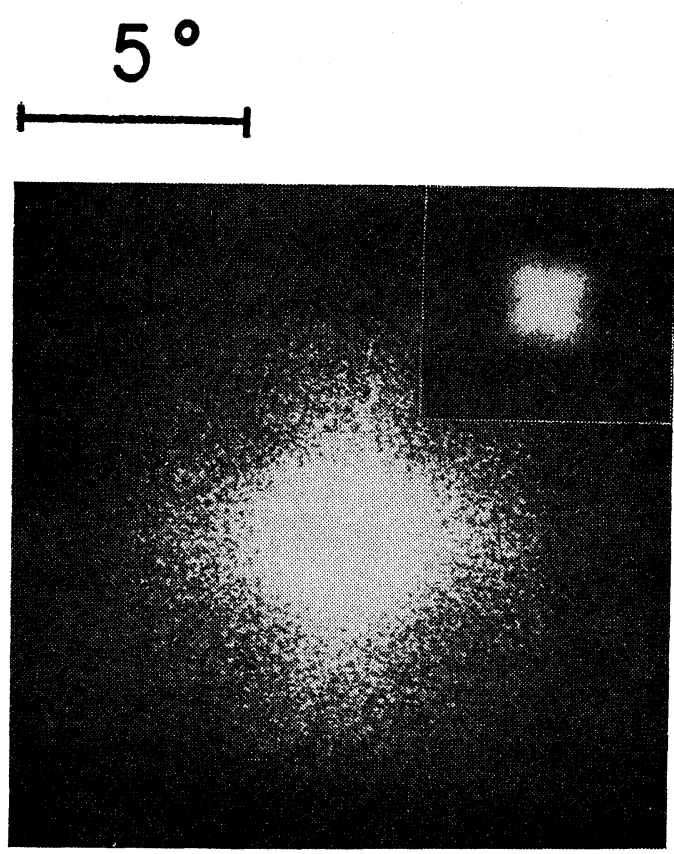

Figure 6. The $H_{\nabla}$ scattering pattern of a polypropylene-polyethylene A-B-type block copolymer film having $20 \mathrm{~mol} \%$ of the ethylene component. The overexposed spherulitic scattering pattern is shown at the upper right corner of the figure. patterns, in terms of $\mu$-dependence, while the fibrils of Model A are shown to give rise to +-type $H_{\mathrm{V}}$ scattering patterns as well as the $\times$ type or circular-type $H_{\mathrm{V}}$ scattering patterns. The +-type scattering pattern in Figure 2 turned out to arise from the fibril of Model A having a value of $\omega_{0}$ nearly equal to $45^{\circ}$. $^{7}$

Scattering patterns, however, occasionally exhibit more complex appearances, as shown in Figures 4-6, than those typical to structures corresponding to Figures 1 and 2. In Figures 4-6, the two types of $H_{\mathrm{V}}$ patterns are simultaneously seen: (i) scattering typical to those from spherulites at small scattering angles and (ii) scattering typical to those from fibrillar structures at higher scattering angles. In this paper we try to show the physical origin of these complex light-scattering patterns.

\section{TEST SPECIMENS AND THE COMPLEX PATTERNS}

Thin films of $c a .30-\mu \mathrm{m}$ thick which exhibit the complex pattern shown in Figure 4 were prepared from poly(tetrafluoroethylene) dispersion. The dispersion was annealed at $480^{\circ} \mathrm{C}$ for 


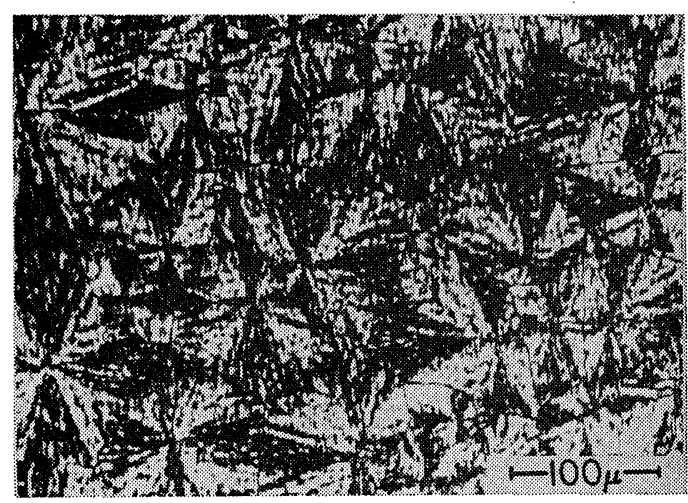

(a)

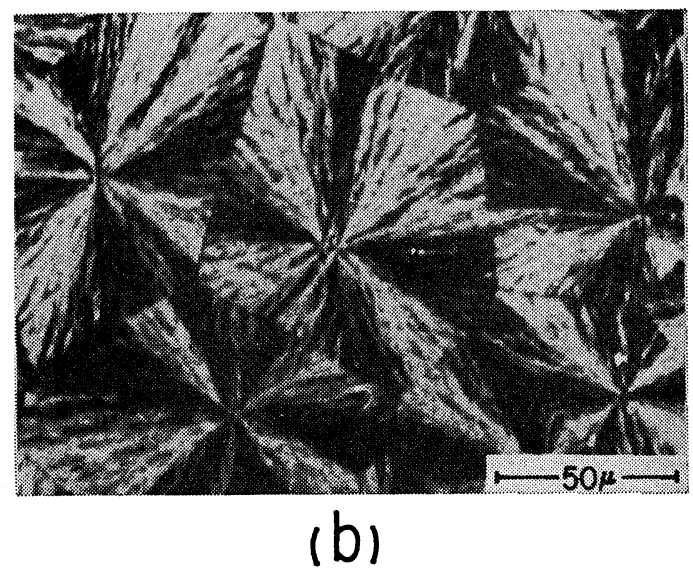

Figure 7. The light micrographs (under crossednicols) of the poly(tetrafluoroethylene) film (a) giving rise to the scattering patterns shown in Figure 4, and the poly(ethylene oxide)-polyisoprene-poly(ethylene oxide) A-B-A block copolymer ${ }^{18}$ film (b) cast from a benzene solution.

$7 \mathrm{hr}$ and subsequently cooled down to room temperature at a rate of $30^{\circ} \mathrm{C} / \mathrm{hr}$. Annealings of the dispersion for a shorter time and/or at a lower temperature give the simple rod-like scattering patterns as shown in Figure 2, in Figure 7 of ref 7, or in Figure 6 of ref 10.

The poly(chlorotrifluoroethylene) films showing the complex pattern (Figure 5) were prepared as follows. The polymer powder was melted at $270^{\circ}$, pressed between laboratory hot plates for $10 \mathrm{~min}$, and subsequently quenched in a water bath at room temperature. The films thus made were put in a silicone-oil bath held at $217^{\circ} \mathrm{C}$ for $10 \mathrm{~min}$, and then rapidly transferred into an another oil bath whose temperature was held at $170^{\circ} \mathrm{C}$ for $40 \mathrm{~min}$ for an isothermal crystallization. The crystallization at lower temperature and for a shorter time gives the simple rod-like scattering patterns as in the case of poly(tetrafluoroethylene) films.

In Figure 6 is shown a typical complex scattering pattern for polypropylene-polyethylene A-B-type block copolymer having $20 \mathrm{~mol} \%$ of the ethylene component. The copolymer was prepared by the Ziegler-Natta method. The powder was preheated at $185^{\circ} \mathrm{C}$ for $15 \mathrm{~min}$, pressed between laboratory hot plates at $185^{\circ} \mathrm{C}$ for $15 \mathrm{~min}$, and subsequently quenched in water at $20^{\circ} \mathrm{C}$. The films thus made were annealed at $125^{\circ} \mathrm{C}$ for $30 \mathrm{~min}$. A similar complex pattern was observed for other A-B-type block copolymers having fractions of ethylene component of 33 and 12 mole percent, and for A-B-A-type block copolymer having a fraction of ethylene component (B) of 13 mole percent. The $V_{\mathrm{V}}$ scattering pattern was circular, as shown in Figure 4(b).

The poly(ethylene oxide)-polyisoprene A-B and $A-B-A$ block copolymers were synthesized according to a method described elsewhere. ${ }^{18} \mathrm{~A}$ complex pattern similar to Figures 4 and 6 was observed for copolymer films having $52 \mathrm{wt} \%$ of poly(ethylene oxide). In Figure $7(b)$ is shown a light micrograph of one of these films.

\section{MORPHOLOGICAL OBSERVATION}

In general there are two ways to interpret the complex scattering pattern. One of them is based upon the possibility that the internal structure of the films is a composite, some regions being composed of large spherulites whose scattering consequently occurs at small angles, and other regions being composed of fibrillar structures of smaller size than that of spherulites, whose scattering therefore occurs at higher angles.

The other way to interpret is based upon the possibility that the structure is uniformly composed of a single entity, i.e., spherulites. In the latter model, the average contour of the spherulite is considered to scatter light at small angles, while internal heterogeneities of the spherulites are considered to scatter light at high angles, in the manner experimentally ob- 
served. A proper choice of the model cannot be made by the light-scattering method alone, but must be made in cooperation with other methods.

The choice was possible in this case by observing the films through a polarizing microscope or electron microscope. It turned out that the internal structure of these test specimens is fully and uniformly composed of impinged spherulites and is not a composite; this is shown, for example, in Figures 7 and 8 . Consequently, the wide-angle scattering typical to the fibrillar structure must be interpreted as arising from the internal heterogeneities of the spherulites correlated over a shorter distance than the size of the spherulite as a whole. The heterogeneities are believed to arise from the existence of the crystalline fibrils, that is, the crystal lamellae or aggregates of the lamellae constituting the spherulites.

In order to explain the complex pattern, the model must satisfy some additional requirements: (i) the fibrils must have a size of the order corresponding to the wide angle scattering, (ii) the fibrils must give the +-type rod-like scattering pattern under $H_{\mathrm{V}}$ polarization, which, in turn, implies that the fibril must have a value of $\omega_{0}$ approximately equal to $45^{\circ}$ and that the fibril must be of the model A fibril, (iii) the fibrils must be oriented more or less parallel to the radial direction of the spherulites, and

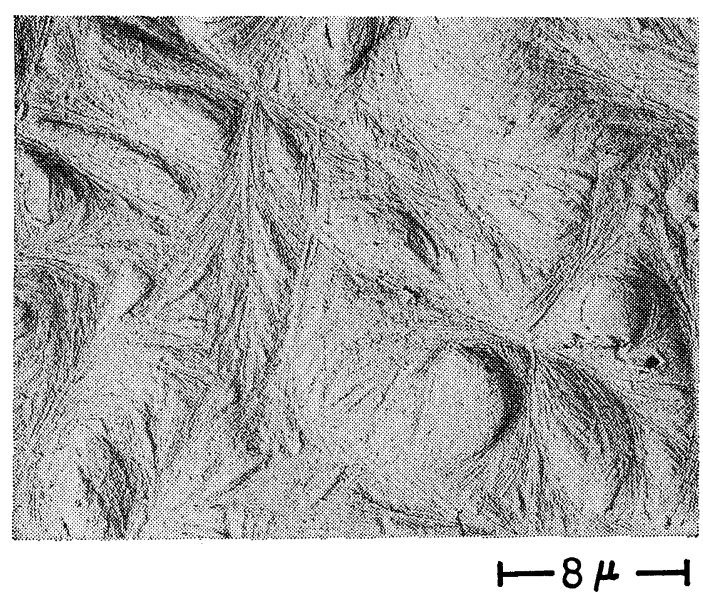

Figure 8. The electron micrograph of the poly(chlorotrifluoroethylene) film which gave rise to the scattering patterns shown in Figure 5. rotation of the fibrils around the spherulite radii must be macroscopically random in order to account for the fact that the orientation of the Maltese cross is parallel to that of the crossed polarizers, and (iv) finally, the spherulites of all the specimens must turn out to have negative birefringence.

\section{MODEL AND CALCULATED RESULTS}

In Figure 9 is shown a schematic diagram of a model which satisfies the above requirements. The crystalline sheets or the fibrils having the chain-axis orientation inclined by the angle $\omega_{0}$ with respect to their bases tend to be aligned in the radial direction of the spherulites. The sheets as a whole are rotated around their axes by the angle $\gamma$. The angle $\gamma$ is macroscopically random but is locally correlated. Thus, according to the fine structure of the spherulites, there are local fluctuations of the optic-axis orientation and refractive indices $n_{1}$ and $n_{2}$ of the uniaxially anisotropic scattering elements.

We shall define angles $\chi$ and $\eta$ as the polar and azimuthal angles of orientation of the optic axis of the scattering element with respect to the spherulite radius. Now the angles $\chi$ and $\eta$ are obviously related to the angles $\omega_{0}$ and $\gamma$ as well as to the angle between the spherulite radius and the major axes of the sheets.

Effects of internal disorder of the spherulites in terms of density, ${ }^{12}$ anisotropy, ${ }^{14}$ and orienta$\operatorname{tion}^{13,15}$ of the optic axis on the spherulitic scattering have been intensively investigated. Such disordered spherulite theories predict that the disorder in the radical direction of the spherulite

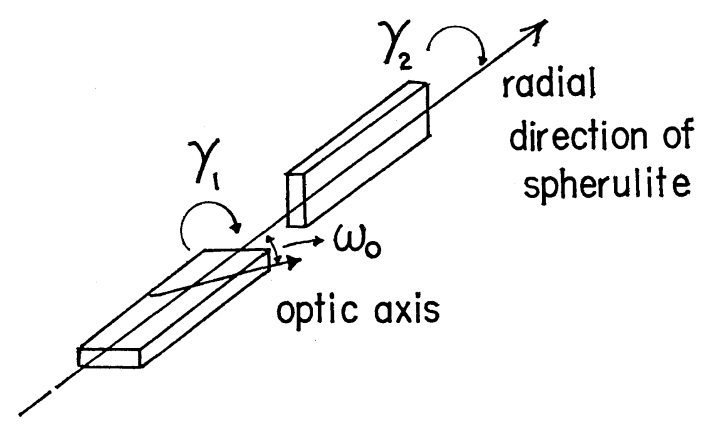

Figure 9. The model of the disordered spherulite. 
is the primary source which gives rise to the experimentally observed excess scattering at $\theta$ greater than $\theta_{\max }$. The excess scattering is shown to depend upon the magnitude of the disorder as well as the correlation distance of the disorder.

In this paper the origin of the complex scattering patterns is proposed in terms of spherulites with radial disorder. The proposal is now considered in more detail.

The $H_{\mathrm{V}}$ scattered intensity of radially disordered spherulites is given by

$$
I_{H_{\mathrm{V}}}=K \cos ^{2} \rho_{2}\left[I_{1} \sin ^{2} 2 \mu+I_{2} \cos ^{2} 2 \mu\right]
$$

where

$$
\begin{aligned}
I_{1}= & \int_{0}^{R} \int_{0}^{R}\left\langle F_{1}\right\rangle_{r_{1}, r_{12}} J_{2}\left(x_{1}\right) J_{2}\left(x_{2}\right) r_{1} r_{2} \mathrm{~d} r_{1} \mathrm{~d} r_{2} \\
I_{2}= & \int_{0}^{R} \int_{0}^{R}\left\langle F_{2}\right\rangle_{r_{1}, r_{12}} J_{2}\left(x_{1}\right) J_{2}\left(x_{2}\right) r_{1} r_{2} \mathrm{~d} r_{1} \mathrm{~d} r_{2} \\
F_{1}= & \delta_{1} \delta_{2}\left(\cos ^{2} \chi_{1}-\sin ^{2} \chi_{1} \cos ^{2} \eta_{1}\right)\left(\cos ^{2} \chi_{2}\right. \\
& \left.\quad-\sin ^{2} \chi_{2} \cos ^{2} \eta_{2}\right) \\
F_{2}= & \delta_{1} \delta_{2} \sin 2 \chi_{1} \sin 2 \chi_{2} \cos \eta_{1} \cos \eta_{2}
\end{aligned}
$$

and $x_{i}=k r_{i} \sin \theta$, where the quantity $k$ is defined by $2 \pi / \lambda$ and $\lambda$ is the wavelength of light in the medium. The quantity $\delta_{i}$ is the birefringence of the uniaxially anisotropic scattering element located at position $r_{i}$ within the spherulites, while the angles $\chi_{i}$ and $\eta_{i}$ are the angles related to the local orientation of the optic axes with respect to the spherulite radius. The quantity $R$ is the radius of the spherulites, and the symbol $\left\langle F_{i}\right\rangle_{r_{1}, r_{12}}$ denotes an average of the quantity $F_{i}$, keeping $r_{1}$ and the separation distance $r_{12}$ constant. The equations are derived by assuming the spherulite to be two-dimensional. The assumption is, however, reasonable for all the specimens, since the specimens are composed of spherulites having a two-dimensional character.

The equations reduce to the special case given by Stein and $\mathrm{Chu},{ }^{13}$ if $\delta_{i}$ and $\chi_{i}$ have constant values of $\delta_{0}$ and $\chi_{0}$ within the spherulites. In this case it follows that

$$
\begin{aligned}
I_{H_{\mathrm{V}}}= & I_{H_{\mathrm{V}}}^{\circ}+K \cos ^{2} \rho_{2}(R / W)^{4}\left[(1 / 8) I_{3} \sin ^{4} \chi_{0} \sin ^{2} 2 \mu\right. \\
& \left.+(1 / 2) I_{4} \sin ^{2} 2 \chi_{0} \cos ^{2} 2 \mu\right]
\end{aligned}
$$

where

$$
I_{3}=\int_{0}^{W} \int_{0}^{W} L\left(x_{12}\right) J_{2}\left(x_{1}\right) J_{2}\left(x_{2}\right) x_{1} x_{2} \mathrm{~d} x_{1} \mathrm{~d} x_{2}
$$

$$
\begin{aligned}
I_{4}= & \int_{0}^{W} \int_{0}^{W} M\left(x_{12}\right) J_{2}\left(x_{1}\right) J_{2}\left(x_{2}\right) x_{1} x_{2} \mathrm{~d} x_{1} \mathrm{~d} x_{2} \\
I_{H_{\mathrm{V}}}^{\circ}= & K \cos ^{2} \rho_{2}\left[\frac{3 \cos ^{2} \chi_{0}-1}{2}\right]^{2} \\
& \times \sin ^{2} 2 \mu\left[2-2 J_{0}(W)-W J_{1}(W)\right]^{2}
\end{aligned}
$$

and $W=k R \sin \theta$. The functions $L\left(x_{12}\right)$ and $M\left(x_{12}\right)$ are the correlation functions for the heterogeneity of the optic-axis twisting-angle $\eta$, and are defined as $L\left(x_{12}\right)=\left\langle\cos 2 \eta_{12}\right\rangle_{x_{12}}$ and $M\left(x_{12}\right)=$ $\left\langle\cos \eta_{12}\right\rangle_{x_{12}}$, where $x_{12}=k r_{12} \sin \theta$ and $\eta_{12}=\eta_{2}-\eta_{1}$.

Equation 6 contains two terms: the first term $\left(I_{H_{V}}^{\circ}\right)$ which gives rise to the four-leaf-clover-type scattering at small angles from a spherulite with random and uncorrelated values of $\eta$, and the second term which gives rise to the wide-angle scattering. Angular dependence of the wideangle scattering with respect to $\mu$ is seen to depend upon the relative contribution of the terms associated with $I_{3}$ and $I_{4}$. It therefore depends upon the value of $\chi_{0}$ and the correlation functions of the optic-axis twisting-angle, $L$ and $M$. Thus the important features of the complex scattering can be, in principle, described by the disordered-spherulite theory of Stein and Chu. ${ }^{13}$

It should be noted here that the average spherulite birefringence $\Delta_{\mathrm{s}}$ is related to the birefringence of the scattering elements $\delta_{0}$.

$$
\Delta_{\mathrm{s}}=\delta_{0}\left(3 \cos ^{2} \chi_{0}-1\right) / 2
$$

The experimental evidence that all the spherulites have negative birefringence suggests that $\cos ^{2} \chi_{0}$ is less than $1 / 3$, i.e., $\chi_{0}$ must be greater than $55^{\circ}$. The $\mu$-dependence of the wide-angle scattering depends upon $\chi_{0}, I_{3}$, and $I_{4}$.

In order to calculate the $H_{\mathrm{V}}$ scattering patterns, the correlation functions $L\left(x_{12}\right)$ and $M\left(x_{12}\right)$ must be known. If the fluctuation of the optic-axis twisting-angle $\eta$ occurs according to the randomwalk statistics of the type discussed by Stein and coworkers, ${ }^{13,16}$ the correlation functions are shown to be given by exponetial functions

and

$$
L\left(x_{12}\right)=\left\langle\cos 2 \omega_{12}\right\rangle_{x_{12}}=\exp \left(-r_{12} / \mathrm{a}\right)
$$

$$
M\left(x_{12}\right)=\left\langle\cos \omega_{12}\right\rangle_{x_{12}}=\exp \left(-r_{12} / 4 a\right)
$$

where $a$ is the correlation distance and depends upon the uncertainty $(\Delta)$ in the twisting angle $\eta$ 


\section{$R / a=10$}

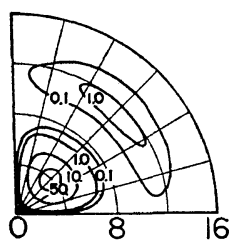

(a)

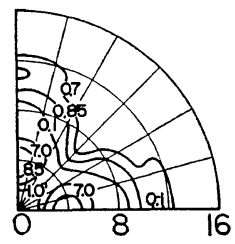

(d)

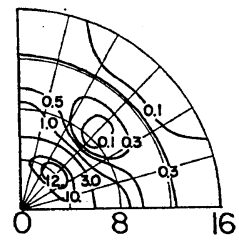

(b)

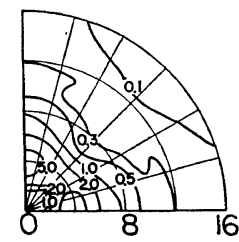

(e)

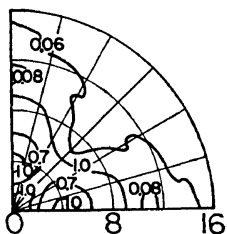

(c)

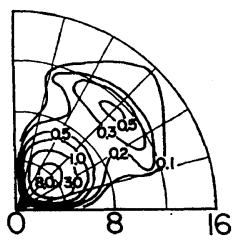

(f)

Figure 10. The calculated $H_{\mathrm{v}}$ scattering patterns for $R / a=100$ and for various values of $\chi_{0}$ : (a) $0^{\circ}$, (b) $30^{\circ}$, (c) $45^{\circ}$, (d) $60^{\circ}$, (e) $65^{\circ}$, and (f) $90^{\circ}$.

\section{$R / a=100$}

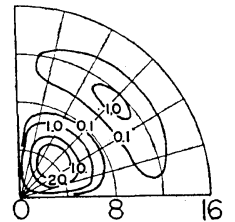

(a)

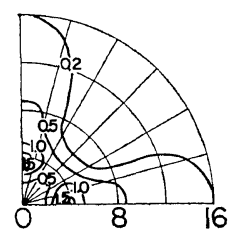

(d)

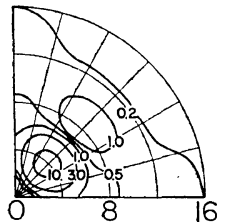

(b)

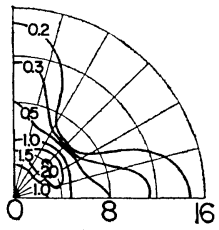

(e)

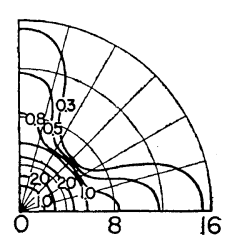

(c)

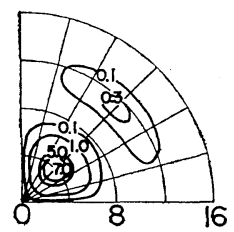

(f)

Figure 11. The calculated $H_{\mathrm{V}}$ scattering patterns for $R / a=10$ and for various values of $\chi_{0}$ : (a) $0^{\circ}$, (b) $30^{\circ}$, (c) $45^{\circ}$, (d) $60^{\circ}$, (e) $65^{\circ}$, and (f) $90^{\circ}$.

of adjacent crystals and upon the size of the crystals (along spherulite radius $l$ ), i.e., $a=l / 2 \dot{\Delta}^{2}$. In this paper, the value of $a$ is given as a simple parameter characterizing the heterogeneity.

In Figures 10 and 11 are shown results of numerically calculated $H_{\nabla}$ patterns for various values of $\chi_{0}$ and $R / a$. As seen in eq 6 , in the case when $\chi_{0}=0^{\circ}$, the $H_{\nabla}$ scatering from the system reduces to the scattering from a perfect spherulite, and therefore there is no effect on the $H_{\mathrm{V}}$ patterns of the disorder in terms of the twisting angle. The primary and secondary scattering maxima are seen at $W \cong 4$ and $W \cong$ 12 (Figures 10(a) and 11(a)). In the case when $\chi_{0}=90^{\circ}$, the term related to $I_{4}$ is zero, so that the wide-angle scattering appears to affect the scattering at $\mu=45^{\circ}$ most, and results in the patterns shown in Figures 10(f) and 11(f). 
On the other hand, in the case when $\chi_{0}$ is $c a$. $45^{\circ}$, the contribution of the term related to $I_{4}$ is predominant over that of the term related to $I_{3}$, so that the wide-angle scattering patterns exhibit the +-type intensity distribution with respect to $\mu$, as seen in Figures 10 and 11 (for values of $\chi_{0}$ being $30^{\circ}, 45^{\circ}, 60^{\circ}$, and $65^{\circ}$ ). If $\chi_{0}$ is equal to $45^{\circ}$ or $60^{\circ}$, the meridional and equatorial small-angle scattering intensities, i.e., the intensities at $\mu=0^{\circ}$ and $90^{\circ}$, and at $W \cong 4.0$, arising from the terms $I_{3}$ and $I_{4}$, become nearly equal to or greater than the small-angle intensity at $\mu=$ $45^{\circ}$, arising from the perfect spherulitic term $\left(I_{H_{\mathrm{V}}}^{\circ}\right)$. Therefore the four-leaf-clover-type small-angle scattering pattern is considerably distorted, as seen in the patterns shown in Figures 10(c), 10(d), $11(\mathrm{c})$, and $11(\mathrm{~d})$. On the other hand, if $\chi_{0}$ is greater than $60^{\circ}$, the intensities associated with the terms $I_{3}$ and $I_{4}$ are smaller than that arising from the term $I_{H_{\mathrm{V}}}^{\circ}$. Therefore the four-leaf-clover-type pattern remains at small $\theta$, as seen in Figure 10(e).

By comparing the experimental and calculated patterns the complex pattern is seen to be best described for a value of $\chi_{0}$ from $65^{\circ}$ to $70^{\circ}$. (see Figure 10(e)). The correlation distance (a) primarily affects the angular distribution of intensities of the extra term with respect to $\theta$. The intensity of the extra term decreases more rapidly for a larger value of $a$ than for a smaller value of $a$. By comparing the calculated patterns with the experimental patterns, it is seen that the value of a of $R / 100$ is more reasonable than $R / 10$.

\section{DISCUSSION}

The radial disorders of anisotropy $\delta^{14}$ and orientation angle $\chi^{13}$ exisiting in spherulites are neglected in the numerical calculations. These do not seem to change the overall contour of the wide-angle scattering, but may slightly modify it according to the extent that the disorders occur. The disorders in angular direction are also neglected in the calculation. These disorders are shown to affect the small-angle scattering, especially the scattering at $\theta$ smaller than $\theta_{\max }$, but to affect the wide-angle scattering only slightly.

By comparing experimental and theoretical patterns, the value of $\chi_{0}$ turned out to be about $65^{\circ}$ to $70^{\circ}$, indicating that the optic axes are oriented at the angle $\chi_{0}$ with respect to the spherulite radius. This may indicate that the chain axes are inclined by the angle of $20^{\circ}$ to $25^{\circ}$ from a direction normal to the bases of crystal lamellae, if the crystalline sheets (stack of crystal lamellae) are aligned along the spherulite radius. This argument assumes that the chain axis is parallel to the optic axis, which is reasonable for the polymers studied in this paper.

In the case of polyethylene spherulites, complex scattering has never been observed for unoriented films. This may be attributed to the fact that the angle $\chi_{0}$ is greater than $c a .70^{\circ}$ and/or that the correlation of $\eta$ persists only for a short distance relative to the spherulites studied here. The correlation distance $a$ is related to the size to the rod-like texture (i.e., a stack of crystal lamellae) constituting the spherulites. The origin of the complex pattern is, therefore, partly associated with the existence of large crystal lamellae which singly or as an aggregate are large enough to give rise to the wide-angle scrttering. The existence of large crystal lamellae is well known for poly(tetrafluoroethylene) and poly(chlorotrifluoroethylene). ${ }^{17}$

The circular-type $V_{\mathrm{V}}$ pattern seen in Figure 4(b) indicates that the density difference between the fibrils and interfibril region is large compared with those shown in Figures 1(b), 2(b), and 5(b).

\section{REFERENCES}

1. R. S. Stein and M. B. Rhodes, J. Appl. Phys., 31, 1873 (1960).

2. R. J. Samuels, J. Polym. Sci., Part A-2, 9, 2165 (1971).

3. S. B. Clough, J. J. van Aartsen, and R. S. Stein, J. Appl. Phys., 36, 3072 (1965).

4. T. Hashimoto, A. Todo, and H. Kawai, J. Polym. Sci., Polym. Phys. Ed., 11, 149 (1973).

5. M. B. Rhodes and R. S. Stein, J. Polymer Sci., Part A-2, 7, 1593 (1969).

6. M. Moritani, N. Hayashi, A. Utsuo, and H. Kawai, Polymer J., 2, 74 (1971).

7. Y. Murakami, N. Hayashi, T. Hashimoto, and H. Kawai, ibid., 4, 452 (1973).

8. J. J. van Aartsen, Eur. Polym. J., 6, 1095 (1970).

9. N. Hayashi and H. Kawai, Polymer J., 3, 140 (1972).

10. T. Hashimoto, Y. Murakami, N. Hayashi, and H. Kawai, ibid., 6, 132 (1932).

11. M. Matsuo, S. Nomura, T. Hashimoto, and H. Kawai, ibid., 6, 151 (1974). 


\section{Origin of a Complex Light-Scattering Pattern}

12. R. S. Stein, P. R. Wilson, and S. N. Stidham, J. Appl. Phys., 34, 46 (1963).

13. R. S. Stein and W. Chu, J. Polym. Sci., Part A-2, 8, 1137 (1970).

14. T. Hashimoto and R. S. Stein, ibid., Part A-2, 9, 1747 (1971).

15. R. S. Stein and T. Hashimoto, ibid., Part A-2,
9, 517 (1971).

16, R. S. Stein and S. N. Stidham, J. Appl. Phys., 35, 42 (1964).

17. P. H. Geil, "Polymer Single Crystals", Intersciences, New York, N.Y., 1963.

18. E. Hirata, T. Ijitsu, T. Soen, T. Hashimoto, and H. Kawai, submitted to Polymer (London). 